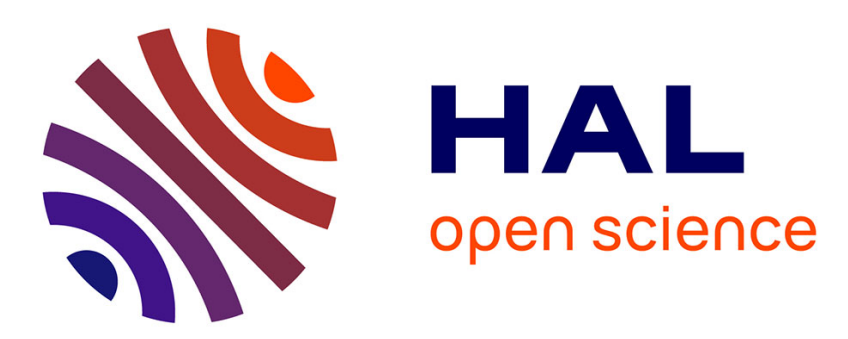

\title{
Fluid simulations of ion scale plasmas with weakly distorted magnetic fields. FLR-Landau fluid simulations
}

Thierry Passot, Pierre Henri, Dimitri Laveder, Pierre-Louis Sulem

\section{To cite this version:}

Thierry Passot, Pierre Henri, Dimitri Laveder, Pierre-Louis Sulem. Fluid simulations of ion scale plasmas with weakly distorted magnetic fields. FLR-Landau fluid simulations. The European Physical Journal D: Atomic, molecular, optical and plasma physics, 2014, 68 (7), 207 (9 p.). 10.1140/epjd/e2014-50160-1 . insu-01352457

\section{HAL Id: insu-01352457 https://hal-insu.archives-ouvertes.fr/insu-01352457}

Submitted on 5 Aug 2020

HAL is a multi-disciplinary open access archive for the deposit and dissemination of scientific research documents, whether they are published or not. The documents may come from teaching and research institutions in France or abroad, or from public or private research centers.
L'archive ouverte pluridisciplinaire HAL, est destinée au dépôt et à la diffusion de documents scientifiques de niveau recherche, publiés ou non, émanant des établissements d'enseignement et de recherche français ou étrangers, des laboratoires publics ou privés. 


\title{
Fluid simulations of ion scale plasmas with weakly distorted magnetic fields
}

\section{FLR-Landau fluid simulations}

\author{
T. Passot ${ }^{1}$, P. Henri ${ }^{1,2}$, D. Laveder ${ }^{1}$ and P.L. Sulem ${ }^{1}$ \\ ${ }^{1}$ UNS, CNRS, Observatoire de la Côte d'Azur, Bd. de l'Observatoire, C.S. 34229, 06304 Nice, France \\ ${ }^{2}$ LPC2E/CNRS 3A Av. de la Recherche Scientifique, 45071 Orlans cedex 2, France
}

Received: date / Revised version: date

\begin{abstract}
Three-dimensional simulations of turbulence in collisionless plasmas are presented, using a fluid model that extends the anisotropic MHD to scales of the order of the ion gyroradius and below in directions perpendicular to the ambient magnetic field. This model, which includes linear Landau damping and finite Larmor radius corrections to all the retained moments, provides an efficient tool to describe Alfvénic turbulence in the absence of cyclotron resonance. When sufficiently small scales are retained, no artificial damping nor collisonal effects is required. Simulations with large-scale Alfvenic driving show the development of power-law spectra with an exponent close to -2.8 for the perpendicular magnetic field at scales smaller than the ion inertial length. The electric field spectrum displays a break at intermediate scales beyond which it approaches a $-1 / 3$ power-law, consistent with Solar Wind observations. These spectra appear in a quasi-stationary state after early-formed sheet-like density and current structures have evolved into filaments. In the presence of temperature anisotropy, the nonlinear development of the mirror instability leads to pressure-balanced magnetic structures surrounded by significant ion velocity fields perpendicular to the ambient field. At later time, the system becomes turbulent, with the disruption of the magnetic structures into parallel filaments.
\end{abstract}

PACS. 5 2.25.Xz, 52.35.Ra, 52.65.Kj, 94.05.Lk, 96.50.Tf

\section{Introduction}

In many instances, space plasmas are collisionless or weakly collisional and turbulent, a regime that can hardly be studied with the usual magnetohydrodynamics (MHD) or its extension including the Hall effect (Hall-MHD), even when permitting ion and electron temperature anisotropies, in part due to a lack of appropriate form of wave damping. A fully kinetic description requiring huge computational ressources, fluid approaches including a modeling of low-frequency kinetic effects such as Landau damping and finite Larmor radius (FLR) corrections at the ion scales, thus appear of special interest to study turbulence in space plasmas.

Landau damping was first retained within a fluid description of a magnetized plasma in [1], where a largescale Landau fluid model was constructed by performing a closure of the moment hierarchy derived from the drift kinetic equation. The fourth-rank cumulants or, in a simplified version, the heat fluxes, are expressed in terms of lower rank moments in a way consistent with the lowfrequency linear kinetic theory, up to the possible replacement of the plasma dispersion function by suitable Padé approximants. Another step was made by supplementing
FLR corrections. This can be performed in two ways. A first approach consists in solving the equations for the non-gyrotropic parts of the pressure or heat flux tensors perturbatively with respect to a small parameter measuring the scale separation between the considered phenomena and the ion gyromotion, both in space and time $[2-$ 4]. Another approach consists in expressing all the nongyrotropic terms directly from the low-frequency kinetic theory, by proceeding as in the closure of the hierarchy $[5$, 6]. In this description, the FLR terms are calculated in a linear approximation within the gyrokinetic scaling, thus retaining quasi-transverse small scales. Concentrating on ion scales, we neglect here the inertia of the electrons, together with their Larmor radius. In the following, we report on this approach and refer to the corresponding model as the FLR-Landau fluid.

The FLR-Landau fluid model includes all the hydrodynamic nonlinearities and is thus suitable for describing the large-scale energy cascade. It also permits the development of temperature anisotropy and the resulting microinstabilities such as the mirror instability, whose growth rate and spectral extension range are accuately reproduced thanks to a precise modeling of the linear Landau damping and FLR corrections to all the retained fluid mo- 
ments. This model accurately reproduces the linear properties of kinetic Alfvén waves (KAWs) such as their dispersion, damping and polarization, in the absence of cyclotron resonance.

The paper is organized as follows. Section 2 briefly describes the main features of the FLR-Landau fluid model and discusses in particular the importance of retaining the magnetic field line distortion in the modeling of Landau damping and FLR corrections in fully nonlinear multidimensional simulations. Section 3 reports on the first three-dimensional numerical simulations using the FLRLandau fluid model, both in the regime of Alfvenic turbulence and in the presence of temperature anisotropy where the mirror instability can occur, leading to the formation of mirror structures and, after their filamentation, to a mirror turbulence regime displaying specific spectral properties. Section 4 summarizes our findings and discusses further developments.

\section{About the FLR-Landau fluid model}

The full system of equations constituting the FLR-Landau fluid model is too complex to be reproduced here due to the lack of space. A few comments on the peculiarities of this model as compared to more common extendedMHD or gyrofluid systems will however be given. The linear properties of the FLR Landau fluid model have been discussed at length in previous papers [5]-[8], showing in particular that the properties of low-frequency modes are accurately captured, except perhaps at intermediate scales where, in some instances, the Padé approximants used in the modelization are the least precise (i.e. when $\left.\zeta=\omega / k_{z} v_{t h}=O(1)\right)$. In addition, and in contrast with gyrofluids, this model does not filter out the fast modes which are in fact well described up to the scale where cyclotron resonance (a phenomenon not captured in fluid formulations) takes place. An interesting point concerns the properties of highly oblique kinetic Alfvén waves (KAWs) which are believed to be the main component of the Alfvén wave cascade at small scales. Above a certain critical angle that decreases as the $\beta$ parameter increases, the KAW polarization becomes right-handed [13-15]. Its frequency can then exceed the ion gyrofrequency without encontering a resonance. It is remarkable that the FLR-Landau fluid model can capture KAW's dispersion relation with a reasonable accuracy even though the frequency lies outside the range of the gyrokinetic approximation [6]. Linear studies of this model also pointed out the importance of taking into account a full description (i.e. in a form that involve Bessel functions of the perpendicular wavenumber) of the non-gyrotropic components of all the retained fluid moments in order to capture the correct KAW eigenvector, even at scales relatively large compared to the ion inertial length. Another remark concerns the importance of the electron Landau damping to account for the correct dissipation of KAWs at scales only slightly smaller than the ion Larmor radius, thus questioning the assumption of isothermal electrons usually made in hybrid methods.
Before discussing first numerical simulations of the multidimensional nonlinear regime, it is of interest to discuss technical points concerning the modelization of the magnetic field line distortion within the kinetic effects.

\subsection{Modeling Landau damping}

We first mention that, as discussed in [1], a proper calculation of Landau damping would involve, at each point, the evaluation of a convolution integral along the magnetic field line attached to this point, a task which is unfeasible on the present day computers. A drastic simplification, consistent with the linear description of the kinetic effects, consists in substituting the local magnetic field line by the straight ambient magnetic field. The calculation of the Landau damping then involves an operator $\mathcal{H}_{z}$ defined as the negative of the Hilbert transform in the direction of the ambient magnetic field (taken in the $z$ direction), which thus reduces in Fourier space to a mere multiplication by $i \operatorname{sgn}\left(k_{z}\right)$. As seen below, this procedure is convenient when the nonlinear problem is considered in one space dimension, but turns out to be inappropriate for the multi-dimensional framework. The dynamical equations for the gyrotropic heat fluxes $q_{\|}$and $q_{\perp}$ indeed involve terms of the form $\nabla \cdot\left(\widetilde{r}_{\perp \|} \widehat{b}\right)$ and $\nabla \cdot\left(\widetilde{r}_{\|\| \|} \widehat{b}\right)$. Here $\widehat{b}$ denotes the unit vector in the direction of the local magnetic field and $\widetilde{r}_{\perp \|}$ and $\widetilde{r}_{\|\|}$two components of the deviation of the gyrotropic fourth order cumulant from their bi-Maxwellian value. Within the present closure, this fourth-order tensor $\widetilde{r}$ includes a contribution proportional to $\mathcal{H}_{z} q$ (here the absence of indices generically stands for the $\perp$ or $\|$ directions). Rewriting

$$
\nabla \cdot(\widetilde{r} \widehat{b})=\widehat{b} \cdot \nabla \widetilde{r}+\widetilde{r} \nabla \cdot \widehat{b}
$$

one sees that it is of interest to examine the well-posedness of the simpler equation (after neglecting all the terms that do not contribute in the linear regime as well as the nonhomogenous ones)

$$
\partial_{t} q-\widehat{b} \cdot \nabla \mathcal{H}_{z} q=0
$$

Let us first show that the one-dimensional case along a prescribed direction making an angle $\alpha$ with the ambient field and parametrized by the abscissa coordinate $\xi$, is well-behaved. One has, in the plane defined by the direction of propagation and the ambient field,

$$
\widehat{b} \cdot \nabla=\left(\widehat{b}_{x} \sin \alpha+\widehat{b}_{z} \cos \alpha\right) \partial_{\xi}=\frac{1}{|B|}\left(B_{x} \sin \alpha+B_{z} \cos \alpha\right) \partial_{\xi}
$$

Furthermore, the zero divergence condition rewrites

$$
\partial_{\xi}\left(\sin \alpha B_{x}+\cos \alpha B_{z}\right)=0
$$

or

$$
\sin \alpha B_{x}+\cos \alpha B_{z}=B_{0} \cos \alpha .
$$

As a consequence

$$
\widehat{b} \cdot \nabla=\frac{B_{0}}{|B|} \cos \alpha \partial_{\xi}
$$


Furthermore, $\mathcal{H}_{z}=\mathcal{H}_{\xi}$, so

$$
\widehat{b} \cdot \nabla \mathcal{H}_{z}=\frac{B_{0}}{|B|} \cos \alpha \partial_{\xi} \mathcal{H}_{\xi}
$$

where in Fourier space the operator $\partial_{\xi} \mathcal{H}_{\xi}$ reduces to the factor $-|k|$, where $k$ is the Fourier variable conjugate to $\xi$. In this special case, the operator thus also rewrites

$$
\widehat{b} \cdot \nabla \mathcal{H}_{z}=\frac{B_{0}}{|B|} \partial_{z} \mathcal{H}_{z}
$$

Apart from the positive prefactor $B_{0} /|B|$, Eq. (2) has the same form as in the purely linear case (i.e. $\partial_{t} q=$ $\left.-\left|k_{z}\right| q\right)$ and is thus well behaved, as could be tested in one-dimensional numerical simulations [10].

Differently, in several dimensions, the term $\widehat{b} \cdot \nabla \mathcal{H}_{z} q$ can lead to a spurious secondary instability in the presence of magnetic field distortion. To analyze the effect of a magnetic field perturbation, it will be more convenient for the sake of simplicity to assume that $\widehat{b}$ is constant, making an angle $\theta$ with the $z$-direction (which in reality can only be true locally). Considering the problem in two space dimensions, one has after making a Fourier transform of Eq. (2)

$$
\partial_{t} q+\left(\widehat{b}_{x} k_{x}+\widehat{b}_{z} k_{z}\right) \frac{k_{z}}{\left|k_{z}\right|} q=0
$$

It is easily seen that when $\lambda \equiv \widehat{b}_{x} k_{x} k_{z} /\left|k_{z}\right|+\widehat{b}_{z}\left|k_{z}\right|<0$, the corresponding Fourier modes are unstable. The growth rate can indeed be rewritten $\lambda=\left(k^{2} /\left|k_{z}\right|\right) \cos \alpha \cos (\alpha-\theta)$. Assuming that the angle $\theta$ is such that $\cos \theta>0$, the condition for instability rewrites $(t-1)(t+1)\left(t^{2}-2 T t-\right.$ $1)<0$, where $t=\tan (\alpha / 2)$ and $T=\tan \theta$. It is seen that for small and positive $\theta$, all wavenumbers for which $t \in\left[-1, T-\sqrt{T^{2}+1}\right]$ or $t \in\left[1, T+\sqrt{T^{2}+1}\right]$ are unstable. This instability can be exemplified numerically by taking an ambient field making a large-scale sinusoidal oscillation along the $z$-direction, with numerical noise on the density field, constant pressures, and zero velocities and heat fluxes. During the growth phase of the instability, the various fields display small-scale oscillations in directions nearly perpendicular to the local magnetic field, in regions where the latter is the most distorted. The above instability, which points out the necessity to accurately compute the direction of the heat flux, could be related to the one found when solving anisotropic diffusion using finite difference schemes that do not preserve monotonicity [11].

The above discussion sugests a subdominant correction of the linear Landau damping terms that ensures wellposedness of the equations. From the previous analysis, it appears important to have a formulation that does not refer to a prescribed direction. The point is that when, in the nonlinear model, the distortion of the ambient field is retained in evaluating the directional derivative $\widehat{b} \cdot \nabla$, it should be so in the negative Hilbert transform (i.e. the operator $\mathcal{H}$ that should also be evaluated along the actual magnetic field lines). In the very special case where the perturbation consists in a global rotation of the ambient field which remains uniform, one has (in Fourier space) $\mathcal{H}=i \widehat{b} \cdot \boldsymbol{k} /|\widehat{b} \cdot \boldsymbol{k}|$, while in the general case $\mathcal{H}$ should be evaluated in the physical space as an integral operator, which, as already mentioned, is presently hardly feasible. At this point, some heuristic modeling is to be used. In order to retain the magnetic line distortion, the procedure used in [17] consists in replacing the denominator of the above formula by a typical (and thus fixed) wavenumber, which up to a multiplicative constant reduces the operator $\mathcal{H}$ to the directional derivative, thus changing the order of the operator and making the Landau damping essentially similar to a collisional dissipation along the magnetic field lines. We suggest an alternative description that preserves the zeroth order of the operator and is exact in the special case of a uniform field, whatever its direction. For this purpose, we write

$$
\mathcal{H}=\frac{\mathcal{F}_{T}(\widehat{b} \cdot \nabla \bullet)}{(\boldsymbol{k} \cdot\langle\boldsymbol{\tau}\rangle \cdot \boldsymbol{k})^{1 / 2}} .
$$

where $\mathcal{F}_{T}$ refers to the Fourier transform and the brakets denote spatial averaging over the whole domain, a description that ensures that the denominator in Eq. (10) is local in Fourier space. Here we denote $\tau_{i j}=\widehat{b}_{i} \widehat{b}_{j}$ and we shall also use $\boldsymbol{n}_{i j}=\delta_{i j}-\widehat{b}_{i} \widehat{b}_{j}$.

\subsection{The non-gyrotropic pressure tensor}

In a similar way, it is also necessary to take into account the magnetic field lines distortion in all the kinetic effects that are evaluated from the linear kinetic theory and retained in the FLR Landau fluid model, in order to prevent the possible development of spurious instabilities in the multi-dimensional nonlinear regime. As an example, we shall explain the procedure that has been adopted for the non-gyrotropic pressure tensor, all the other FLR corrections being treated in a similar way.

The idea is to replace the quantities evaluated from the linear kinetic theory by expressions that remain intrinsic whatever the direction of the magnetic field and reduce to the primitive quantities in the linear asymptotics. by

We are thus led to replace Eqs. (33) and (38) from [5]

$$
\nabla \cdot \boldsymbol{\Pi}=\nabla \cdot\left[-\mathcal{A} \boldsymbol{n}-\mathcal{B} \boldsymbol{\epsilon} \cdot \widehat{b}+\widehat{b} \otimes \boldsymbol{\Pi}_{\|}+\boldsymbol{\Pi}_{\|} \otimes \widehat{b}\right]
$$

where $\boldsymbol{\epsilon}$ denotes the fundamental antisymetric 3rd-rank tensor and

$$
\boldsymbol{\Pi}_{\|}=-\boldsymbol{n} \cdot \nabla \mathcal{C}+\boldsymbol{\epsilon} \cdot \widehat{b} \cdot \nabla \mathcal{D} .
$$

It is then necessary to modify the "potentials" $\mathcal{A}, \mathcal{B}, \mathcal{C}$, $\mathcal{D}$ accordingly, writing intrinsic formulas which are equivalent when neglecting the magnetic field line distortion. As obvious examples, one is led to replace $\partial_{z}$ by $\widehat{b} \cdot \nabla$ and, for a vector field $\boldsymbol{V}$ that does not include a mean value of order unity, $\nabla_{\perp} \cdot \boldsymbol{V}_{\perp}$ by $\boldsymbol{n}: \nabla \boldsymbol{V}$.

Note that there is no unique way of writing an "intrinsic" formulation starting from linear kinetic formulas. 
A comparison with the exact large-scale nonlinear asymptotic expressions ([4] and references therein) nevertheless provides useful guidelines [18].

\section{Numerical simulations}

The FLR-Landau fluid model is numerically integrated using a Fourier spectral method in a 3D periodic domain with a physical extension five times larger in the parallel than in the perpendicular ones in order to focus on the quasi-transverse dynamics. A resolution of $128^{3}$ grid points is used, leading to resolve lenghts of the order of a fraction of the ion gyroradius in the perpendicular directions. Dealiasing is performed by putting to zero all the fields Fourier components outside a cube of side $2 k_{\max } / 3$ for the Alfvenic turbulence runs (Section 3.1) and $k_{\max } / 2$ for the simulations of the mirror instability (Section 3.2). The time integration is performed with an explicit 3rdorder Runge-Kutta scheme, which imposes strong stability constraints on the timestep due to the presence of the electron physics in the Landau damping that must be resolved. In all the simulations, no extra dissipation nor filtering is used, the damping provided by the ion and electron Landau resonances being sufficient to absorb the energy transferred to small scales by the nonlinear cascade.

\subsection{Alfvén wave turbulence}

A simple procedure to generate a turbulence of kinetic Alfvén waves (KAWs) is to drive the system by a random forcing supplemented in the velocity equation, in the form

$$
F_{i}(t, \mathbf{x})=\sum_{1<n<N} F_{i, n}^{0} \cos \left(\omega_{K A W}\left(\boldsymbol{k}_{n}\right) t-\boldsymbol{k}_{n} \cdot \boldsymbol{x}+\phi_{i, n}\right)
$$

for different wavevectors $\boldsymbol{k}_{n}$, with $F_{i, n}^{0}$ and $\phi_{i, n}$ being the amplitude and phase of the $n^{\text {th }}$ mode forming the $i^{\text {th }}$ component of the external driver $F_{i}$. When taking for $\omega_{K A W}(\boldsymbol{k})$ the KAW frequency at wavevector $\boldsymbol{k}$, the system will generate KAWs by resonance at this frequency. KAW frequencies are calculated from the linearized FLRLandau fluid system, using a symbolic mathematics software. The dispersion relation is tabulated and used as an input in the FLR-Landau fluid code. Eight KAWs (four forward and four backward propagating waves with an orthogonal polarization) making an angle of 80 degrees with the ambient magnetic field are excited at the largest scale. The driving is turned on (resp. off) when the sum of the kinetic and magnetic energies is below (resp. above) a certain threshold taken such that the typical magnetic field fluctuation does not exceed $20 \%$ of the ambient field magnitude. Here we assume initially equal isotropic ion and electron temperatures with $\beta=1$.

Interestingly, for these parameters, electron Landau damping plays an important role in the damping of Alfvén waves, even though the smallest considered scale in the simulation is not smaller than a tenth of the ion gyroradius. This conclusion is reached by comparing two freely

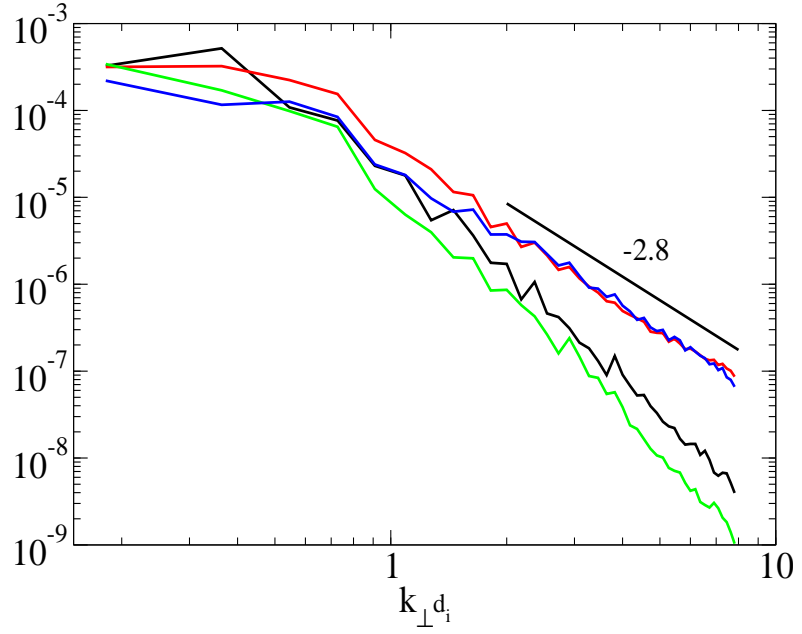

Fig. 1. Angle-averaged energy spectra as functions of the perpendicular wavenumber (in units of $d_{i}^{-1}$ ) for $k_{z}=0$, of the perpendicular magnetic field and ion velocity at $t=500$ (black and green lines respectively), together with their average (red and blue lines respectively) over the time interval $600<t<700$.

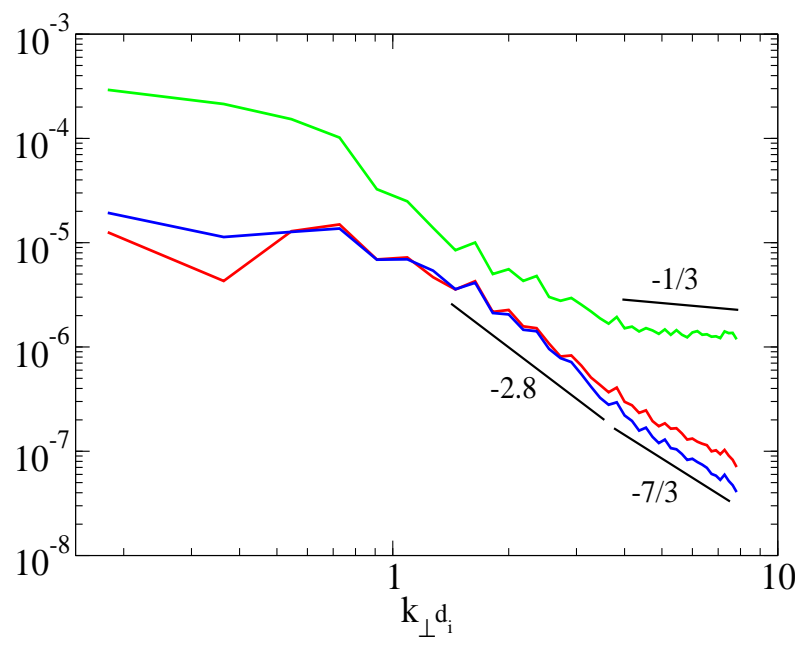

Fig. 2. Angle-averaged energy spectra as functions of the perpendicular wavenumber (in units of $d_{i}^{-1}$ ) for $k_{z}=0$, of the number density (red line), of the parallel magnetic field fluctuation (blue line) and of the perpendicular electric field (green line) averaged over the interval $600<t<700$.

decaying simulations, one with the full model and the other one using a quasi-normal closure for the electron dynamics (zero fourth rank cumulants, and thus no Landau damping), taking as initial condition a well-developed turbulent state generated with the full model, using Alfvén wave forcing. While the former model continues to evolve in a smooth way, the run where the electron Landau damping is turned off rapidly generates a spurious accumulation of energy at small scales and has to be stopped.

Another interesting point concerns the role of the nongyrotropic contributions. Simulations performed without these terms (but still keeping the Hall electric field and Landau dampings) showed some differences with the full model that will not be discussed here, but clearly demonstrate 
that the no-FLR model can also be simulated without extra dissipation nor filtering, beyond ion and electron Landau dampings.

We now discuss a few results of a run which was integrated in a box of parallel and transversal sizes $L_{\|}=$ $196 d_{i}$ (here $d_{i}$ holds for the ion inertial length) and $L_{\perp}=$ $34.6 d_{i}$, during 700 inverse ion cyclotron frequencies $\Omega_{i}^{-1}$ with a time step equal to $\Delta t=2 \cdot 10^{-3} \Omega_{i}^{-1}$. After $t=$ 600 , the situation is statistically stationary. The angleaveraged transverse magnetic and velocity field spectra, are displayed in Fig. 1 as functions of $k_{\perp}$ for $k_{z}=0$, at time $t=500$ and after averaging over time between $t=600$ and $t=700$. While the parallel spectrum is very steep (not shown), in agreement with the fact that the KAW cascade should be anisotropic and mostly in the perpendicular directions, a clear power-law behavior with a slope close to -2.8 is observed in the transverse directions at the late times, for a range of wavenumbers extending from $k_{\perp} d_{i}=2$ to the end of the numerical zone (cutting off the range associated with dealiasing). In particular, in contrast with simulations involving Laplacian-type viscous effects, no dissipative zone is observed. In the purely perpendicular direction, dissipation is mediated by nonlinear coupling which drains energy to oblique directions where it can dissipate through Landau damping. Moreover, Landau damping cannot be viewed as working mostly within a small-scale dissipation range. The existence of a proper inertial range, free of injection and dissipation, can thus be questioned in this kind of systems. The fact that the simulation does not show any spurious accumulation of energy at small scales in fact indicates that, within this power-law range, energy must be dissipated. Even though our model does not contain a parametrization of the small scales, its behavior is somewhat reminiscent of the so-called Large Eddy Simulations [19] where power-law spectra also develop until the smallest retained scale.

The slope of the perpendicular electric field spectrum (shown in Fig. 2 as averaged on the time interval $600<$ $t<700$ ) displays, for $k_{\perp} d_{i}>4$, a clear power law with a $-1 / 3$ slope, associated with a $-7 / 3$ range of the parallel magnetic field spectrum. This shallower part is also visible on the density spectrum, but not on the perpendicular magnetic field, as previously seen. Preliminary analysis seems to link this part of the spectrum with sharp gradients of $B_{z}$ in transverse directions, possibly associated with magnetosonic waves. The slopes of the perpendicular magnetic spectrum are close to those observed in the Solar Wind $[20,21]$ in the so-called dissipation range, where the spectral exponents range from -2.5 to -2.8 , separated from the usual Kolmogorov inertial range by a transition zone where the slope can be much steeper. The magnetic and electric spectra observed in the present simulations compare also very well with those observed in gyrokinetic simulations [22].

The transition between the steeper spectrum observed at $t=500$ and the quasi-stationary spectra observed later is associated with a clear change in the topology of the typical density structures, from sheets (Fig. 3 left) to filaments (Fig. 3 right) aligned with the local magnetic field.
These panels actually display data that have been filtered by removing Fourier modes with $k>k_{\max } / 2$, in order to suppress a small-scale noise that does not affect the dynamics but is associated to the existence of powerlaw spectra extending down to the smallest scale. These spectra are indeed the Fourier signature of physical fields which are either just continuous or for which only the lowest order derivatives are finite. This transition seems nevertheless associated to a breakup of the sheets rather than to a roll up, with a later pile up of the structures (see Fig. 4 showing a density cut in a plane perpendicular to the ambient field at times $t=500$ and $t=600$ ).

Such filamentary structures are also observed at the level of the electric current density in isotropic incompressible Hall-MHD [23] and in electron MHD turbulence [24] where they are shown to be due to the Hall term, standard MHD rather leading to sheet-like structures. Magnetic spectra close to $k^{-8 / 3}$ (thus significantly steeper that the usual $k^{-7 / 3}$ spectra obtained for KAW [25], whistler [26] or Hall-MHD [27] turbulence on the base of phenomenological arguments, but which are somewhat closer to the ones observed in our FLR-Landau fluid simulations), are observed in the electron MHD simulations of [24] and also in simulations of a simple nonlinear fluid model for KAWs [28], where the density structures are nevertheless observed to be sheets. Interestingly, the existence of filaments or sheets in a KAW model has also been shown to depend on the ratio of diffusive to resistive dampings [29]. In the present simulations, resistive damping is absent. Further study is necessary to identify the physical mechanisms at the origin of this transition between sheets to filaments, and in particular the role of resistivity due to collisional effects.

The current density also undergoes a transition towards filamentary structures. It is shown in Fig. 5 at time $t=500$ where sheets (in dark yellow) are still visible. Magnetic field lines (in green) are also plotted to illustrate their typical distortion. A few isosurfaces of the density field, taken close to their minimal (in blue) and maximal (in red) values are also plotted to show that they take the form of elongated filamentary structures following the field lines.

The ion velocity field is quasi two-dimensional, as shown in Fig. 6 (top panel) which also shows density isosurfaces at time $t=550$, with a line of sight almost perpendicular to the ambient magnetic field. In contrast, the electron velocity streamlines (Fig. 6, middle panel) have a more prononced three-dimensional character, with large parallel velocities in localized regions (right part of the panel). The bottom panel of Fig. 6 displays the ion velocity streamlines together with density isosurfaces viewed at a small angle from the direction of the ambient field. A small density region (top right) is seen in the center of a vortex, while a sheet-like structure (top left) appears to be stretched between two co-rotating vortices.

A diagnosis that is often used in the analysis of satellite data is the so-called magnetic compressibility spectrum, given by the ratio of the longitudinal to the total magnetic field spectra as a function of the transverse wavenumber. 

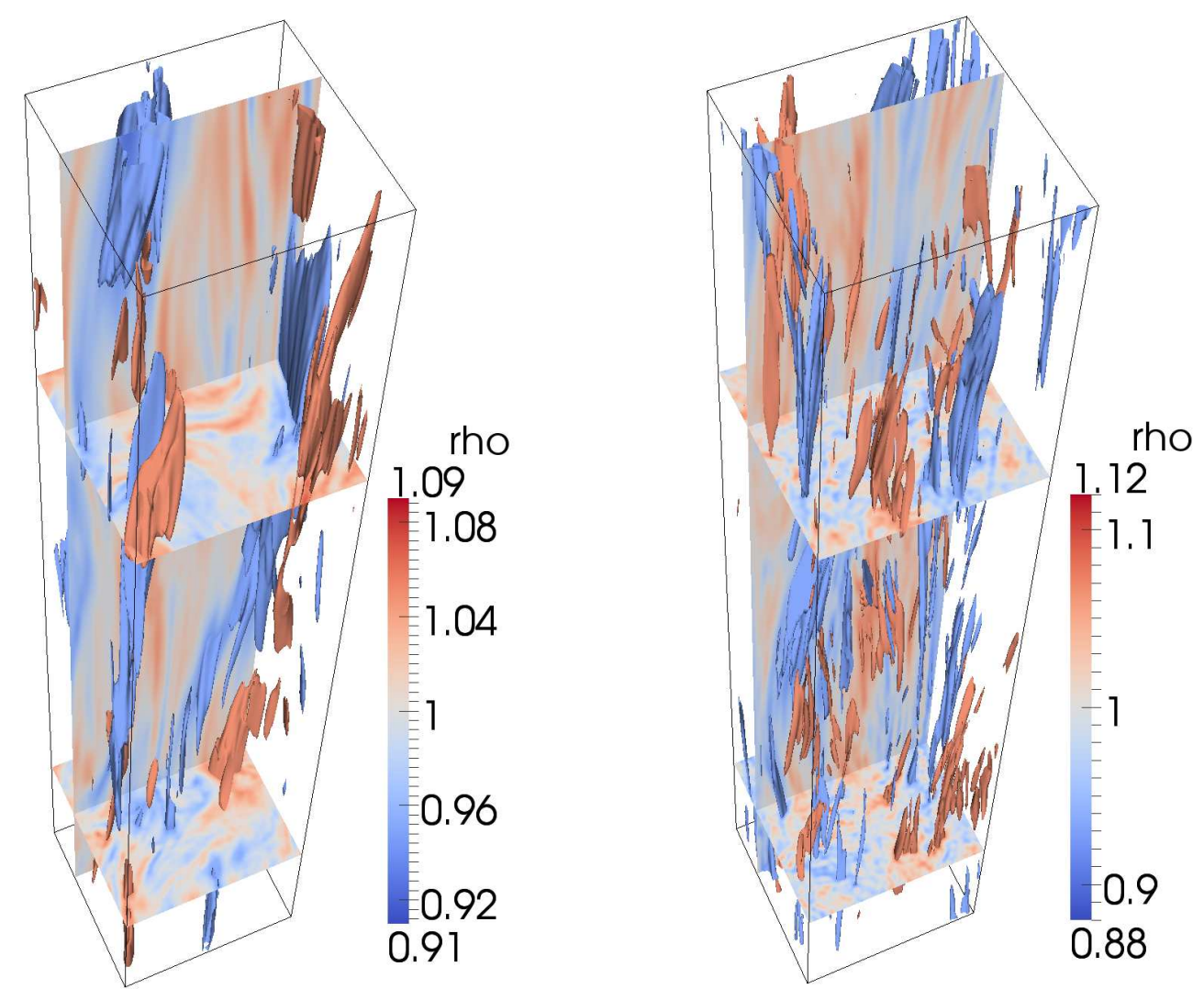

Fig. 3. Isosurfaces at $55 \%$ of the minimum (blue) and $55 \%$ of the maximum (orange) of the density field at $t=500$ (left) and $t=600$ (right) together with color-scale plots in parallel and transverse planes. Minimum and maximum values are indicated on the color bars.
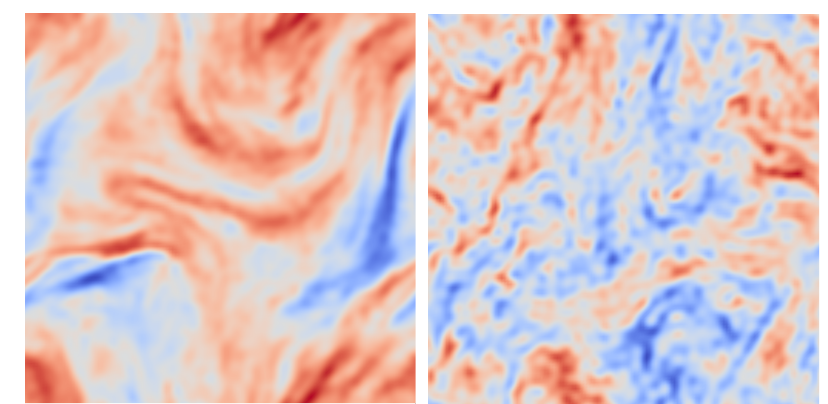

Fig. 4. Cross sections of the density field in the upper transverse planes displayed in the left and right panels of Fig. 3

This quantity, averaged over the time interval $600<t<$ 700 , is shown for the above simulation in Fig. 7 (black line). A clear signature of KAWs is observed, with a small value at large scales that increases around $k d_{i}=1$ and saturates at values of about 0.4 at small scales, consistent with the linear kinetic theory [30].

\subsection{Formation of mirror structures and development of mirror turbulence}

This FLR-Landau fluid model can also be used to address plasma dynamics in the presence of temperature anisotropy. In this section, we illustrate this issue with the development and the nonlinear saturation of the mirror instability, taking $\beta_{\| p}=2, T_{\perp p} / T_{\| p}=2, T_{\perp e} / T_{\| e}=1$ and $T_{\| e} / T_{\| p}=1$. The size and aspect ratio of the simulation boxes is chosen so that the mode $(1,1)$ is mirror unstable, leading to the formation of 4 mirror structures. In this simulation, $L_{\perp}=17.6 d_{i}, L_{\|}=35.1 d_{i}$ and the timestep is $\Delta t=1.25 \cdot 10^{-3} \Omega_{i}^{-1}$.

The simulation is initiated with small random noise on a uniform density, with zero velocity, uniform magnetic field and pressures and zero heat fluxes. After a phase of linear growth, the mirror instability saturates, creating magnetic field structures in the form of elongated cigarshaped maxima, and minima with a more complex topology, in the form of saddles (see Fig. 8, left). As predicted by the linear theory, anti-correlation between magnetic intensity and perpendicular ion pressure is clearly observed (not shown). Anti-correlation with density is also visible in Fig. (8) (left), although in a less obvious way. Interestingly, we observe the presence of quasi two-dimensional ion velocity fields (red-yellow lines) surrounding the magnetic structures, with maximal velocities of the order of $0.2 v_{A}$. This regime contrasts with the ordering performed in theoretical developments near the mirror instability threshold, where the velocity field is found to be subdominant in a weakly nonlinear asymptotics [31]. In any realistic situ- 


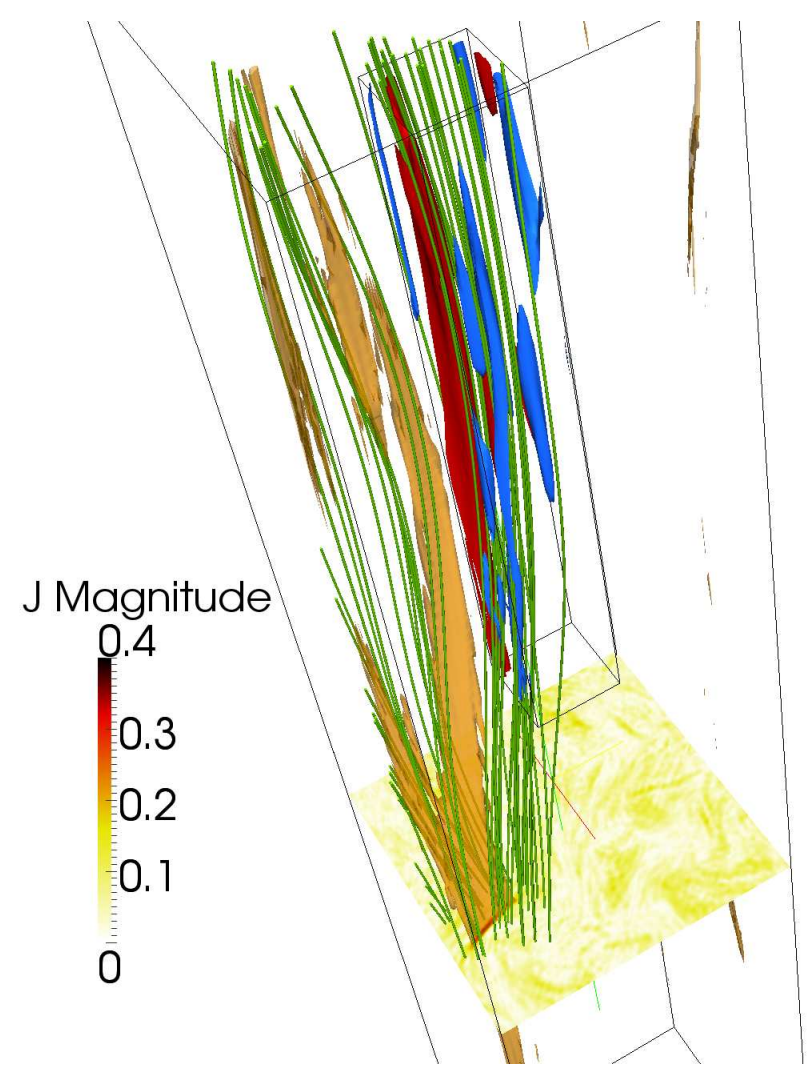

Fig. 5. Isosurfaces at $t=500$ of the current density at $50 \%$ of its maximum value (dark yellow), together with those of the density (restricted in a sub-domain) at $\rho=0.98$ (blue) and at $\rho=1.023$ (red). Magnetic fields lines (in green) are superimposed, indicating that the structures tend to align with the magnetic field.

ation, the deviation to threshold can hardly be so small as to ensure a quasi-static evolution. It is thus of interest to investigate the further evolution of these mirror structures. We show in Fig. 8 (right), a later view (using the same angle of sight) of the density and magnetic fields, together with two cross-sections of the $z$-component of the vorticity. The density maxima have evolved in the form of thin sheets in the strain field of two conter-rotating vortices. The velocity field has also developed significant parallel components, as can be seen in the (red-yellow) stream lines spiraling along a vortex. The isosurfaces of the magnetic intensity for values close to its maximum display ripples which ultimately lead to the formation of filaments, elongated along the magnetic field, which has relaxed to a much straighter form. The magnetic fluctuations are at this stage indeed mostly parallel, as can also be seen from the magnetic compressibility spectrum (Fig. 7, red line) averaged over the time interval $402.5<t<450$. The turbulence that develops in this simulation is thus significantly different from that resulting from Alfvenic driving and should be identifiable in space plasma observations in regions (e.g. behing a shock wave) where the temperature anisotropy can exceed the threshold of the mirror instability. The question arises whether the turbulence regime displayed in this simulation is related to the
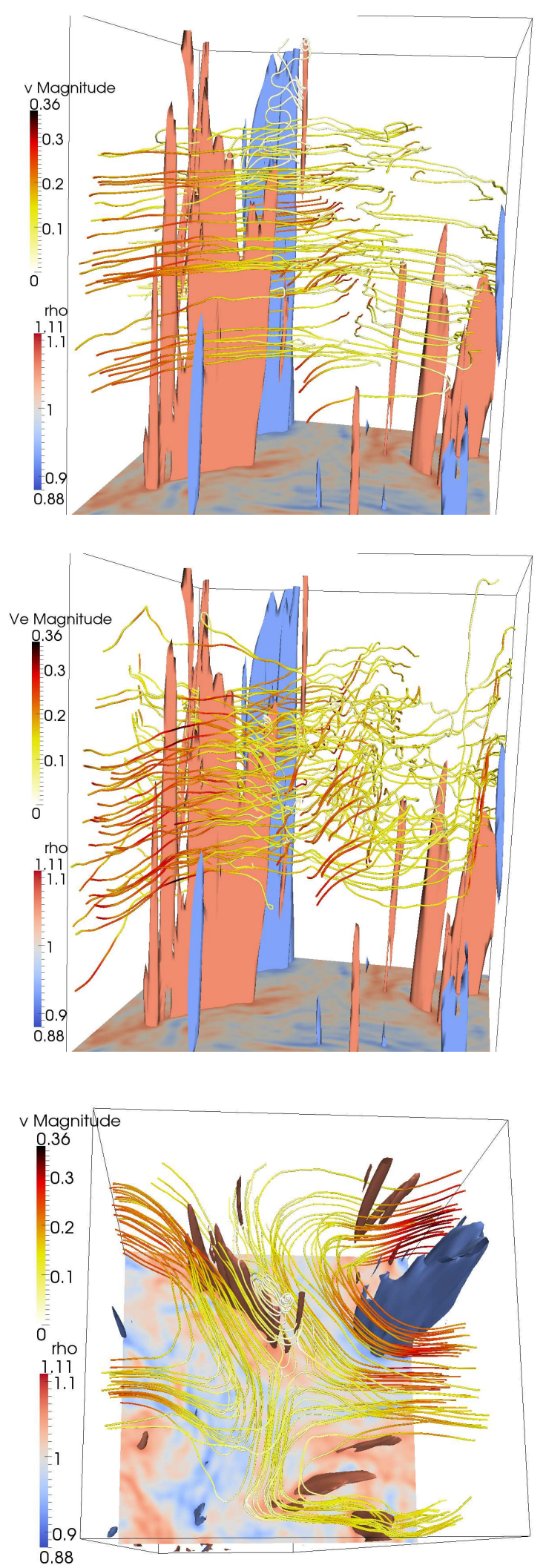

Fig. 6. Ion (top) and electron (middle) velocity field lines together with isosurfaces of large (red) and small (blue) density fluctuations at $t=550$. The bottom panel displays in a view from above the ion velocity showing vortical motions around density minima and stretching of large density regions between co-rotating vortices. 


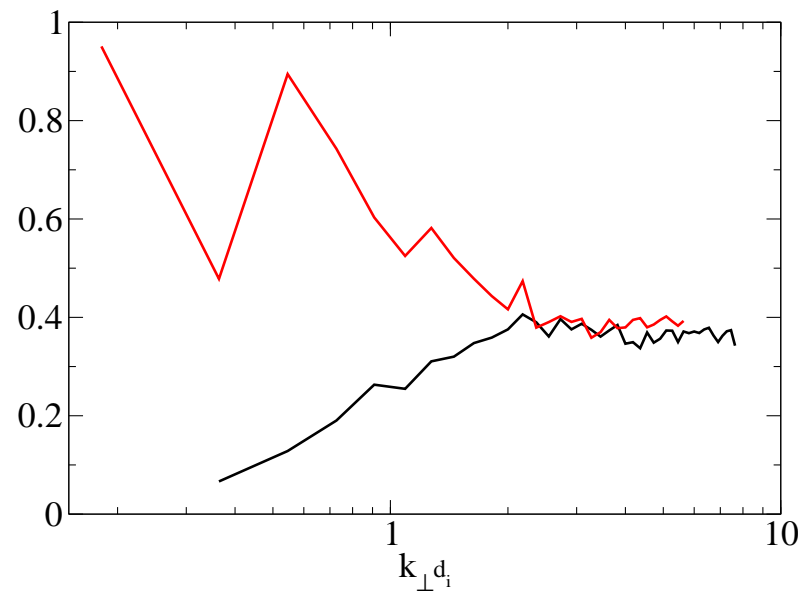

Fig. 7. Magnetic compressibility spectrum as a function of perpendicular wavenumber (in units of $d_{i}^{-1}$ ) of the developed regime of Alfvenic (black line) and mirror (red line) turbulences.

turbulence seen in the terrestrail magnetosheath where new power-law spectra, not predicted by the usual turbulence theories, have been reported [32].

\section{Conclusion}

The preliminary results presented in this paper demonstrate the capability of the FLR-Landau fluid model, which supplements a linear description of the low-frequency kinetic effects to the usual anisotropic Hall-MHD, to simulate turbulence in collisionless plasmas, in the close subionic range where important dynamical phenomena were revealed by satellite observations in the Solar Wind or the terrestrial magnetosheath. In the case of Alfvenic turbulence, the magnetic fluctuations display power-law spectra with exponents consistent with Solar Wind observations [21] and gyrokinetic simulations [22], in spite of the moderate resolution $\left(128^{3}\right)$ of the present simulations. Indeed, retaining ion and electron Landau dampings turns out to be sufficient to stabilize the numerical scheme and to permit the extension of the self-similar spectral range up to the largest retained wavenumber. It is in particular not necessary to include an artificial damping which, for a given resolution, significantly reduces the extension of the spectral range effectively simulated. Furthermore, in the presence of temperature anisotropy, the accurate description of linear Landau damping and FLR corrections enables the simulation of the mirror instability and of its nonlinear saturation. On a longer time-scale, the previously formed pressure balanced structures around which the existence of a significant, mostly two-dimensional ion velocity field was observed, get distorted, leading to the development of a turbulence regime which strongly differs from Alfvenic turbulence, in particular at the level of the spectrum of magnetic compressibility. Another important result concerns the role of the electron Landau damping at the ion scale when $\beta$ is of order unity, an observation which may question the assumption of isothermal electrons usually made in hybrid simulations.

When compared with fully kinetic simulations in situations where the distribution functions remain close to bi-Maxwellian, fluid models display some interesting aspects in spite of the involved approximations. They are not only less demanding in computational power, but are also much more flexible, for example at the level of the permitted initial conditions and driving processes. Furthermore, as the various terms in the equations can easily be switched off and on, they often lead to easier physical interpretations, allowing for an easy estimate of the various contributions to a given phenomenon. Another advantage of fluid models compared to particle in cell (PIC) or hybrid PIC methods concerns their low level of numerical noise. Investigation of small-scale turbulence is only possible if the noise level is sufficiently low compared to the turbulence energy level, a situation that could require a prohibitively large number of particles per cell in PIC simulations.

As already mentioned, the present model retains the FLR effects at the linear level only, as the result of an estimate of the nongyrotropic moments by a closure procedure consistent with the low-frequency linear kinetic theory. Differently, fully nonlinear expressions of these quantities are available in the literature, at least to leading order within a large-scale asymptotics. Further developments should include a mixed description of the FLR effects, involving a matching between the large-scale nonlinear description and the present kinetic-based closure at smaller scale, an approach which may be of special interest for example when addressing questions such as stochastic the heating of the plasma.

The research leading to these results has received funding from the European Commission's Seventh Framework Programme (FP7/2007-2013) under the grant agreement SHOCK (project number 284515). This work has been performed using the computing facilities provided by the "Mesocentre SIGAMM" hosted by Observatoire de la Côte d'Azur and using high-performance computing resources from GENCI- IDRIS (Grant i2013047042).

\section{References}

1. Snyder PB, Hammett GW and Dorland W Phys. Plasmas 4 (1997) 3974-3985

2. Macmahon A Phys. Fluids 8 (1965) 1840-1845

3. Goswami P, Passot T and Sulem PL Phys. Plasmas 12 (2005) 102109

4. Ramos JJ Phys. Plasmas 12 (2005) 052102

5. Passot T and Sulem PL Phys. Plasmas 14 (2007) 082502

6. Passot T, Sulem PL and Hunana P Phys. Plasmas 19 (2012) 082113

7. Hunana P, Goldstein ML, Passot T, Sulem PL, Laveder D and Zank GP Proc. Solar Wind 13, AIP Conference Proceedings, 1539 (2013) 179-182

8. Hunana P, Goldstein ML, Passot T, Sulem PL, Laveder D and Zank GP Astrophys. J. 766 (2013) 93

9. Laveder D, Passot T and Sulem PL Ann. Geophys. 31 (2013) 1195-1204 

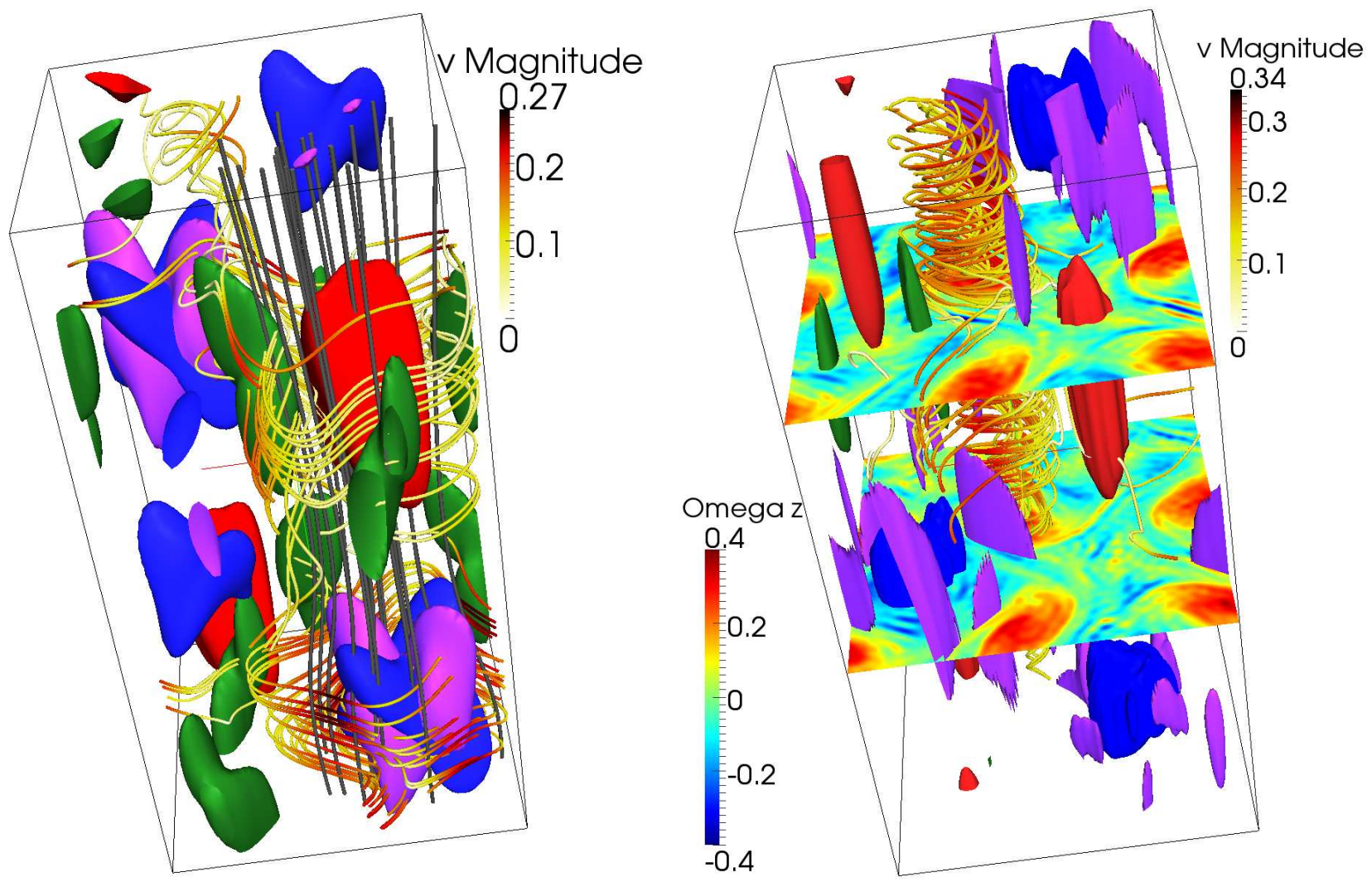

Fig. 8. Isosurfaces of magnetic field amplitude [large positive (negative) fluctuations are indicated in red (blue)] together with those of the density [large positive (negative) fluctuations are indicated in purple (green)] at $t=390$ (left panel) and $t=425$ (right panel). Superimposed are the ion velocity and (on the left panel) the magnetic field lines (in grey). The right panel also displays the parallel vorticity in two transverse planes. The choice of isosurface levels is as follows: in the left panel, red (blue) levels are taken at $4 / 5(2 / 3)$ of the maximum (1.31) [minimum (0.63)], purple (green) levels correspond to $2 / 3(4 / 5)$ of the maximum (1.09) [minimum (0.94)]; in the right panel, red (blue) levels are taken at 3/4 (2/3) of the maximum (1.17) [minimum $(0.70)]$, purple (green) levels correspond to $2 / 3(3 / 4)$ of the maximum (1.08) [minimum (0.88)].

10. Laveder D, Marradi L, Passot T and Sulem PL Geophys. Res. Lett. 38 (2011) L17108

11. Sharma P and Hammett GW J. Comput. Phys. 227 (2007) 123

12. Hunana P, Laveder D, Passot T, Sulem PL and Borgogno D Astrophys. J. 743 (2011) 128

13. Gary SP J. Plasma Phys. 35 (1986) 431

14. Belmont G and Rezeau L Ann. Geophys. 5 (1987) 59

15. Sahraoui F, Belmont G and Goldstein ML Atrophys J. 748 (2012) 100

16. Dorland W and Hammett GW Phys. Fluids B 5 (1993) 812

17. Sharma P, Hammett GW, Quataert E and Stone JA Atrophys J. 637 (2006) 952

18. Passot T and Sulem PL, in preparation for J. Plasma Phys. (2014).

19. Lesieur M and Metais O Ann. Rev. Fluid Mech. 28 (1996) 45

20. Sahraoui F, Goldstein ML, Robert P and Khotyaintsev YV, Phys. Rev. Lett. 102 (2009) 231102

21. Sahraoui F, Goldstein ML, Belmont G, Canu P and Rezeau L, Phys. Rev. Lett. 105 (2010) 131101

22. Howes GG, TenBarge JM, Dorland W, Quataert E, Schekochihin AA, Numata R and Tatsuno T Phys. Rev. Lett. 107 (2011) 035004
23. Miura H and Araki K, J. Phys. Conf. Series 318 (2011) 072032

24. Meyrand R and Galtier S Phys. Rev. Lett. 111 (2013) 264501

25. Schekochihin AA, Cowley SC, Dorland W, Hammett GW, Howes GG, Quataert E and Tatsuno T Astrophys. J. Suppl. Series 182 (2009) 310-377

26. Biskamp D, Schwartz E, Zeiler A, Celani A and Drake JF Phys. Plasmas 6 (1999) 751

27. Galtier S Phys. Rev. E 77 (2008) 015302

28. Boldyrev S, Horaites K, Xia Q and Perez JC, Astrophys. J. $\mathbf{7 7 7}$ (2013) 41

29. Smith KW and Terry PW Astrophys. J. 730 (2011) 133

30. Sahraoui F, Belmont G and Goldstein ML Astrophys. J. $\mathbf{7 4 8}(2012) 100$

31. Califano F, Hellinger P, Kuznetsov E, Passot T, Sulem PL and Trávníček PM J. Geophys. Res. 113 A08219

32. Sahraoui F, Belmont G, Rezeau L, Cornilleau-Wehrlin N, Pinçon JL and Balogh A Phys. Rev. Lett. 96 (2006) 075002 